OPEN ACCESS

Edited by:

Renee Lay Hong Lim,

UCSI University, Malaysia

Reviewed by:

Qixia Luo,

Zhejiang University, China Jon William Weeks, United States Food and Drug Administration, United States

${ }^{*}$ Correspondence:

Mehri Haeil

m.haeili@tabrizu.ac.ir; m.haeili@yahoo.com Hanieh Mohajjel Shoja mohajelh@yahoo.com

Specialty section: This article was submitted to Antimicrobials, Resistance and Chemotherapy, a section of the journa

Frontiers in Microbiology

Received: 28 April 2021

Accepted: 09 July 2021

Published: 05 August 2021

Citation:

Moghimi M, Haeili M and

Mohaijel Shoja H (2021)

Characterization of Tigecycline Resistance Among Tigecycline

Non-susceptible Klebsiella pneumoniae Isolates From Humans, Food-Producing Animals, and in vitro

Selection Assay.

Front. Microbiol. 12:702006. doi: 10.3389/fmicb.2021.702006

\section{Characterization of Tigecycline Resistance Among Tigecycline Non-susceptible Klebsiella pneumoniae Isolates From Humans, Food-Producing Animals, and in vitro Selection Assay}

\author{
Mohaddeseh Moghimi ${ }^{1}$, Mehri Haeili* and Hanieh Mohajjel Shoja ${ }^{2 *}$
}

'Department of Animal Biology, Faculty of Natural Sciences, University of Tabriz, Tabriz, Iran, ${ }^{2}$ Department of Plant Biology, Faculty of Natural Sciences, University of Tabriz, Tabriz, Iran

Emergence of extensively drug-resistant isolates of Klebsiella pneumoniae has prompted increased reliance on the last-resort antibiotics such as tigecycline (TGC) for treating infections caused by these pathogens. Consumption of human antibiotics in the food production industry has been found to contribute to the current antibiotic resistance crisis. In the current study, we aimed to investigate the mechanisms of TGC resistance among 18 TGC-non-susceptible (resistant or intermediate) K. pneumoniae (TGC-NSKP) isolates obtained from human $(n=5)$, food animals $(n=7)$, and in vitro selection experiment $(n=6)$. Isolates were genotyped by multilocus sequence typing (MLST). ramR, acrR, rpsJ, tet $A$, and $\operatorname{mgrB}$ (for colistin resistance) genes were sequenced. The presence of tet $X$, tet $X 1$, and carbapenemase genes was examined by PCR. Susceptibility to different classes of antibiotics was evaluated by disc diffusion and broth macrodilution methods. The expression level of acrB was quantified by RT-qPCR assay. The 12 TGC-NSKP isolates [minimum inhibitory concentrations (MICs) $=4$ $32 \mathrm{mg} / \mathrm{l}]$ belonged to 10 distinct sequence types including ST37 $(n=2)$, ST11, ST15, ST45, ST1326 (animal isolates); ST147 $(n=2$, human and animal isolates); and ST16, ST377, ST893, and ST2935 (human isolates). Co-resistance to TGC and colistin was identified among 57 and $40 \%$ of animal and human isolates, respectively. All human TGC-NSKP isolates carried carbapenemase genes (bla $\mathrm{OXA}_{-48}$, bla $\mathrm{NDM}_{\mathrm{N}-1}$, and bla NDM -5). tetX/X1 genes were not detected in any isolates. About $83 \%$ of TGC-NSKP isolates $(n=15)$ carried ramR and/or acrR alterations including missense/nonsense mutations (A19V, L44Q, I141T, G180D, A28T, R114L, T119S, Y59stop, and Q122stop), insertions (positions +205 and +343), or deletions (position +205) for ramR, and R90G substitution or frameshift mutations for acrR. In one isolate ramR amplicon was not detected using all primers used in this study. Among seven colistin-resistant isolates, five harbored inactivated/mutated MgrB due to premature termination by nonsense mutations, insertion of IS elements, and frameshift mutations. All isolates 
revealed wild-type RpsJ and TetA (if present). Increased expression of acrB gene was detected among all resistant isolates, with the in vitro selected mutants showing the highest values. A combination of RamR and AcrR alterations was involved in TGC non-susceptibility in the majority of studied isolates.

Keywords: tigecycline resistance, Klebsiella pneumoniae, food animals, ramR, AcrR, AcrAB efflux pump

\section{INTRODUCTION}

Antibiotic resistance is rising to dangerously high levels in both human and veterinary medicine. The limited number of antibiotics coming to the market and new threats arising from extensively drug-resistant bacteria bring us perilously to the end of antibiotic era in which common treatable infections and minor injuries can once again be fatal. The use of human antibiotics in food animals as growth promoters or prophylactic agents has been identified as a significant contributing factor to increasing antimicrobial resistance (Van et al., 2020). Food animals have the potential to serve as reservoir for antibiotic-resistant bacteria, which can be transmitted to humans via direct contact or the food chain (Founou et al., 2016). Klebsiella pneumoniae is among the most problematic pathogens that have acquired much public health concern due to developing resistance to most clinically important antibiotics. It is an opportunistic pathogen that commonly causes a variety of the community- and hospitalacquired infections including urinary tract infection, pneumonia, and bloodstream infection. The emergence and dissemination of carbapenemase-producing strains of $K$. pneumoniae in healthcare facilities constitute a serious threat to public health (Pitout et al., 2015). The management of infections caused by carbapenemresistant $K$. pneumoniae (CRKP) is complicated and often requires the use of last-resort antibiotics such as tigecycline (TGC) and colistin (Renteria et al., 2014; Sader et al., 2015). TGC is the first member of the novel class of glycylcyclines with expanded-spectrum antibacterial activity against Gram-negative and Gram-positive bacteria. It is approved by the Food and Drug Administration (FDA) for use in complicated skin and skin structure infections, complicated intra-abdominal infections, and community-acquired bacterial pneumonia (Beabout et al., 2015). TGC acts by inhibition of bacterial protein synthesis and has the ability to evade the classical mechanisms mediating resistance to tetracyclines including ribosomal protection and active efflux mediated by Tet proteins (Livermore, 2005).

In $K$. pneumoniae, TGC resistance is being increasingly reported since its approval (Spanu et al., 2012; Chiu et al., 2017a). TGC resistance in K. pneumoniae is believed to be mainly mediated by the overexpression of AcrAB efflux pump, which is regulated by local repressor AcrR and transcriptional activator RamA. The latter protein is also negatively regulated by RamR whose mutations have been found to contribute to significant increases in $\operatorname{ram} A$ and subsequently acr $A B$ expression upon resistance occurrence (Hentschke et al., 2010; Chiu et al., 2017b). Decreased susceptibility to TGC has been also found to be related to alterations in the efflux pump encoding gene tetA (Du et al., 2018), or rpsJ (Beabout et al., 2015), the gene that encodes the ribosomal S10 protein. Moreover, enzymatic inactivation by TetX protein, a flavin-dependent monooxygenase, has been described to confer TGC resistance among some clinical pathogens (Moore et al., 2005; Deng et al., 2014). We aimed in the current study to investigate the TGC resistance determinants from a diverse group of TGC-non-susceptible (intermediate or resistant) K. pneumoniae (TGC-NSKP) isolates of clinical and animal origins and in vitro developed TGC-NSKP mutants. Also, the sequence types of TGC-NSKP isolates were determined by multilocus sequence typing (MLST) to identify major types associated with TGC non-susceptibility in each group of bacterial isolates from different host origins.

\section{MATERIALS AND METHODS}

\section{Bacterial Isolates}

Klebsiella pneumoniae isolates from animal and human sources were included in this study. Animal isolates were obtained by taking cloacal swabs (using sterile cotton swab) from randomly selected broilers at a major chicken slaughterhouse. Taken samples were seeded on Eosin Methylene Blue (EMB) agar plates and were incubated at $37^{\circ} \mathrm{C}$ for $24 \mathrm{~h}$. Screening for TGC-nonsusceptible isolates among the grown colonies on EMB agar was performed using Mueller-Hinton broth (MHB) supplemented with $3 \mathrm{mg} / \mathrm{l}$ of TGC according to the method described in our previous study (Pishnian et al., 2019). Moreover, five clinical TGC-NSKP isolates obtained from patients hospitalized in two teaching hospitals of the country were included as human isolates. Identification of isolates was performed by conventional biochemical methods (Mahon et al., 2018).

\section{In vitro Selection of Tigecycline-Resistant Bacteria}

To identify the genetic alterations mediating TGC resistance, upon TGC exposure, an in vitro resistance induction experiment was performed by exposing three TGC-susceptible (TGC-S) isolates to elevated concentrations of TGC. The MuellerHinton agar (MHA) plates supplemented with a sub-inhibitory concentration of antibiotic $[1 / 2 \times$ of minimum inhibitory concentrations (MICs)] were inoculated with $3 \times 10^{5} \mathrm{CFU} / \mathrm{ml}$ TGC-S bacterial suspension and were incubated for 24-72 h. Colonies appearing on each plate were randomly picked and reisolated on media with the same concentration of TGC or concentrations, which were within $1.25-1.5 \times$ of previous concentration. In cases where no growth was observed at higher concentrations, colonies appeared at the same concentration were picked and transferred to MHA supplemented with the same and higher concentration of TGC. The obtained in vitro 
induced TGC-NSKP isolates were subjected to antimicrobial susceptibility testing.

\section{Antimicrobial Susceptibility Testing}

The MICs of TGC (Glentham Life Sciences, Corsham, United Kingdom) (batch nos. 389SOI and 081GRB), colistin (colistin sulfate, Glentham Life Sciences, United Kingdom; batch no. 844WZQ), and imipenem (IPM) (Glentham Life Sciences, United Kingdom; batch no. 205WLC) were determined by broth macrodilution method using freshly prepared (less than 12-h-old) MHB from Difco (BD Diagnostic Systems, Sparks, MD, United States). The susceptibility to other classes of antibiotics was determined by disc diffusion method (KirbyBauer) according to the Clinical and Laboratory Standards Institute (CLSI) guidelines using the following antibiotics: gentamicin, amikacin, ampicillin, ceftriaxone, cefepime, nalidixic acid, ciprofloxacin, levofloxacin, gatifloxacin, tetracycline, doxycycline, minocycline, chloramphenicol, nitrofurantoin, and fosfomycin (BBL Sensi-Disc ${ }^{\text {TM }}$, Becton-Dickinson, Sparks, $\mathrm{MD}$, United States). Due to the lack of established CLSI breakpoints for TGC at this time, the FDA breakpoints issued for Enterobacteriaceae (susceptible $\leq 2 \mathrm{mg} / \mathrm{l}$, intermediate $=4 \mathrm{mg} / \mathrm{l}$, and resistant $\geq 8 \mathrm{mg} / \mathrm{l}$ ) were applied for interpretation of results. Isolates characterized with colistin MIC values greater than $2 \mathrm{mg} / \mathrm{l}$ were categorized as resistant according to guidelines described by the European Committee on Antimicrobial Susceptibility Testing (EUCAST). Escherichia coli ATCC 25922 was used as a quality-control strain for antimicrobial susceptibility testing.

\section{Bacterial Genotyping by Multilocus Sequence Typing}

MLST with seven housekeeping genes ( $r p o B, g a p A, m d h$, pgi, phoE, infB, and ton $B$ ) was carried out for TGC-NSKP isolates of human and animal origins following the methods described previously (Diancourt et al., 2005). The allelic profiles and sequence types (STs) were assigned by using the K. pneumoniae MLST database provided by the Institut Pasteur, Paris, France. ${ }^{1}$

\section{Molecular Determinants of Tigecycline, Colistin, and Carbapenem Resistance}

The TGC-NSKP isolates were screened for the presence of tet $X$ and tet $X 1$ genes by performing PCR with gene-specific primers (Table 1) [a second pair of primers were used for detection of tet $X 1$ gene (forward 5'-GCGACATTCCTGAACCAGAAACG and reverse $5^{\prime}$-CGGACGATTACTCTTCCAAGG)]. The coding regions of $r a m R, a c r R, r p s j$, tet $A$, and $m g r B$ [for colistin (Col) resistance] were amplified and sequenced using the primers listed in Table 1. For isolates that did not yield a PCR product using primers targeting amplification of $\mathrm{ram} R$ coding sequence as well as some flanking regions (ramR-ext), amplification with other pairs of primers targeting an internal region of ramR coding sequence was repeated (ramR-int). Mutations were characterized by comparing the sequences with those of K. pneumoniae ATCC 700603 and three TGC-susceptible isolates (TGC MICs $=0.25 \mathrm{mg} / \mathrm{l}$ ). The impact of the identified amino acid substitutions on the biological function of the protein

${ }^{1}$ http://bigsdb.pasteur.fr/klebsiella/klebsiella.html

TABLE 1 | Nucleotide sequences of primers used in this study.

\begin{tabular}{|c|c|c|c|}
\hline Primer name & Sequence $\left(5^{\prime}-3^{\prime}\right)$ & Size of product (bp) & References \\
\hline ramR-ext-F & TGGTCAGACGTGCCAAGATC & 654 & This study \\
\hline ramR-int-F & GCAAGCGTTACTGGAAGCTG & \multirow[t]{2}{*}{515} & \multirow[t]{2}{*}{ This study } \\
\hline ramR-int-R & CAAAGCCAAGGGCGATAATCT & & \\
\hline acrR-F & GTAAAGTCATTAACCTATGGCACG & 667 & This study \\
\hline rpsj-F & CAATCGTAATGGGTATGAGGAGT & \multirow[t]{2}{*}{514} & \multirow[t]{2}{*}{ This study } \\
\hline rpsj-R & CCTGAGTAACACGGTTTGCTT & & \\
\hline tetA-F & ACCCAACAGACCCCTGATCGT & \multirow[t]{2}{*}{1,133} & \multirow[t]{2}{*}{ This study } \\
\hline tetA-R & GCAAGTAGAGGGCAGCGCCT & & \\
\hline tetX-F & TTAGCCTTACCAATGGGTGT & \multirow[t]{2}{*}{243} & \multirow[t]{2}{*}{ Bartha et al., 2011} \\
\hline tetX-R & CAAATCTGCTGTTTCACTCG & & \\
\hline mgrB-R & GGCGTGATTITGACACGAACAC & 347 & Haeili et al., 2017 \\
\hline \multicolumn{4}{|l|}{ RT-qPCR } \\
\hline acrB-F & CAGCTTAACGCCTCGATCATC & \multirow[t]{2}{*}{127} & \multirow[t]{2}{*}{ This study } \\
\hline acrB-R & CCAGCTCAATITTGGCGACATC & & \\
\hline rpsl-F & CCGTGGCGGTCGTGTTAAAGA & \multirow[t]{2}{*}{109} & \multirow[t]{2}{*}{ Cannatelli et al., 2013} \\
\hline rpsl-R & GCCGTACTTGGAGCGAGCCTG & & \\
\hline
\end{tabular}


(i.e., neutral or deleterious) were further predicted by using the Protein Variation Effect Analyzer tool (PROVEAN) (Choi and Chan, 2015). The IS Finder database ${ }^{2}$ was used to identify and analyze insertion sequences. Moreover, the presence of carbapenemase-encoding genes (bla $a_{\mathrm{KPC}}, b l a_{\mathrm{VIM}}, b l a_{\mathrm{NDM}}$, and bla $\left.a_{\mathrm{OXA}-48}\right)$ were examined by PCR using the primers and amplification conditions described previously (Poirel et al., 2011). The nucleotide sequences of $b l a_{\mathrm{NDM}}$ were determined using the primers NDM-F-5'-GCCCAATATTATGCACCCGGTC and NDM-R-5'-AGCGCAGCTTGTCGGCCAT (Jafari et al., 2019).

\section{Assessment of acrB Expression}

To investigate the association between TGC non-susceptibility and overexpression of AcrAB efflux pump, the expression level of $a c r B$ gene was measured using RT-qPCR analysis. The total RNA from all TGC-S and TGC-NS bacterial cells was harvested using a GeneAll RiboEx Total RNA extraction kit (GeneAll Biotechnology, Seoul, South Korea). cDNA was synthesized from $1 \mu \mathrm{g}$ of RNase-free DNase I (Takara Biotechnology, Dalian, China)-treated total RNA using Revert Aid first-strand cDNA synthesis kit (Thermo Fisher Scientific, Waltham, MA, United States). Real-time PCR amplification was performed using a Power SYBR green PCR master mix (Applied Biosystems, Foster City, CA, United States) on a Eco Real-Time PCR system (Illumina, San Diego, CA, United States). The relative gene expression levels were calculated using the $2^{-\Delta \Delta C T}$ formula with rpsL housekeeping gene as internal control. A TGC-S isolate with TGC MIC of $0.25 \mathrm{mg} / \mathrm{l}$ was used as a reference strain. In the case of in vitro selected mutants, expression levels of acrB were compared with those of parental TGC-S isolates.

\section{Nucleotide Sequence Accession Numbers}

The nucleotide sequences of the studied genes have been deposited at GenBank nucleotide sequence database under the following accession numbers:

MW653710 to MW653712 (mutated/altered mgrB), MW653713 and MW653714 (wild-type $m g r B$ ), MW653715 to MW653725 (mutated/altered $\mathrm{ramR}$ ), MW653726 to MW653730 (wild-type $\mathrm{ramR}$ ) MW653731 to MW653736 (mutated/altered acrR), MW653737 (HK10-S, AK88-S, AK298, AK299, and HK98), MW653738 (HK2-S), MW653739 (AK294 and AK297) (wild-type acrR), MW653740 (rpsJ, all isolates had identical sequences), and MW653741 (tet $A$, all isolates had identical sequences).

\section{RESULTS}

\section{Bacterial Isolates, Molecular Typing, and Antimicrobial Susceptibility Testing}

Among the 1,430 samples taken from healthy broilers (collected from 83 different farms), six TGC-resistant (TGC-R) bacteria were detected, all corresponding to K. pneumoniae. The TGC$\mathrm{R}$ K. pneumoniae (TGC-RKP) isolates displayed TGC MICs

${ }^{2}$ https://isfinder.biotoul.fr ranging from 8 to $32 \mathrm{mg} / \mathrm{l}$. Moreover, one TGC-R isolate (AK513, TGC MIC $=8 \mathrm{mg} / \mathrm{l}$ ) was included from our previous work (Pishnian et al., 2019), which was obtained from a turkey during the screening for colistin-resistant bacteria (Table 2). The seven TGC-R animal isolates belonged to six different sequence types including ST37 ( $n=2$ isolates) and ST11, ST15. ST45, ST147, and ST1326 ( $n=1$ each). Testing susceptibility to other antimicrobials revealed that TGC resistance was linked to a multidrug-resistant phenotype, and all animal TGC-RKP isolates showed resistance to quinolones, chloramphenicol, other members of tetracycline family and nitrofurantoin. The full resistance rate to gentamycin, ceftriaxone, and fosfomycin was found to be $42.8,28$, and $28 \%$, respectively. One isolate, AK294, was found to be extendedspectrum $\beta$-lactamase (ESBL) producer using the combination disc method. Co-resistance to colistin and TGC was observed among $57 \%$ of animal isolates belonging to ST37, ST11, and ST15. All TGC-R animal isolates were susceptible to amikacin and IPM. Among the five studied human isolates, one was TGC-R $(\mathrm{MIC}=8 \mathrm{mg} / \mathrm{l})$, and the remaining isolates showed intermediate susceptibility to TGC (MICs $=4 \mathrm{mg} / \mathrm{l})$. The MLST distributed the five TGC-NSKP human isolates into five distinct sequence types, including ST16, ST147 (also found in one animal isolate), ST377, ST893, and ST2935. Two human isolates showed simultaneous resistance to TGC (nonsusceptible), colistin, and IPM, with the remaining three isolates being characterized with co-resistance to IPM and TGC. Fosfomycin and amikacin were among the few antimicrobials that showed $40 \%$ activity against the TGC, IPM, and \pm Col NSKP human isolates. The antimicrobial susceptibility profiles of the studied isolates are described in Table 2.

\section{In vitro Selection of Klebsiella pneumoniae Mutants With Reduced Susceptibility to Tigecycline}

Three TGC-susceptible K. pneumoniae isolates (TGC MICs $=0.25 \mathrm{mg} / \mathrm{l}$ ) were exposed to increasing concentrations of TGC. As $50 \%$ of the TGC-NSKP isolates in this study were characterized with co-resistance to colistin and TGC, we included one TGC-S but Col- $\mathrm{R}$ isolate among the in vitro selected isolates to see if resistance to colistin facilitates development of TGC resistance. Overall, it took 21-23 selection cycles to obtain six TGC-NSKP isolates with TGC MICs of 32 ( $n=1$ mutant), 16 ( $n=3$ mutants), 8 ( $n=1$ mutant), and $4 \mathrm{mg} / \mathrm{l}(n=1$ mutant $)$ from three parental TGC-S isolates (Table 2). There was no significant difference between the selection cycles required to reach a TGC-NSKP isolate among the ColR and ColS isolates. Interestingly, all progenitor TGC-S isolates that were susceptible to tetracycline, minocycline, doxycycline, nalidixic acid, and chloramphenicol before induction became fully resistant (or showed intermediate susceptibility) to these antibiotics upon TGC non-susceptibility induction.

\section{Detection and Sequence Analysis of Resistance Conferring Genes}

The plasmid-encoded tet $X$ and tet $X 1$ genes were not detected in any of the TGC-NSKP isolates of both origins. All TGC-NSKP isolates of human, animal, and in vitro selected origins revealed 
TABLE 2 | Genotypic and phenotypic characteristics of tigecycline susceptible and non-susceptible K. pneumoniae isolates obtained from human, food animals, and in vitro selection assay.

\begin{tabular}{|c|c|c|c|c|c|c|c|c|c|c|c|c|}
\hline \multirow{2}{*}{ Isolate } & \multirow{2}{*}{$\begin{array}{l}\text { Sequence } \\
\text { type }\end{array}$} & \multicolumn{3}{|c|}{ MIC (mg/l) } & \multirow[t]{2}{*}{ RamR } & \multirow[t]{2}{*}{ AcrR } & \multirow[t]{2}{*}{ TetA } & \multirow[t]{2}{*}{ MgrB } & \multirow[t]{2}{*}{ Carbapenemase } & \multicolumn{3}{|c|}{ Antimicrobial susceptibility profile } \\
\hline & & $\operatorname{Tgc}$ & Col & Ipm & & & & & & $\mathbf{s}$ & $\mathbf{I}$ & $\mathbf{R}$ \\
\hline \multicolumn{13}{|c|}{ Wild type isolates } \\
\hline AK88-S & ND & 0.25 & $>128$ & 0.25 & WT & WT & - & WT & - & $\begin{array}{l}\text { D, LVX, NA, TE, GM, Ml, } \\
\text { CRO, AN, GAT, F/M, FOS, } \\
\text { C, CIP, FEP }\end{array}$ & & AM \\
\hline HK2-S & ND & 0.25 & 0.25 & 1 & WT & WT & - & WT & - & AN, FOS, TE, D, Ml & & $\begin{array}{c}\text { AM, CRO, F/M, NA, } \\
\text { FEP, GM, CIP, LVX, } \\
\text { GAT, C }\end{array}$ \\
\hline \multicolumn{13}{|c|}{ Laboratory induced mutants } \\
\hline HK10-R1 & ND & 32 & 0.25 & 0.25 & WT & $\begin{array}{c}\Delta 14 \mathrm{nt} \\
\text { (g58-t71) } \\
\text { (frameshift) }\end{array}$ & - & WT & - & $\begin{array}{l}\text { LVX, GM, CRO, AN, GAT, } \\
\text { F/M, FOS, CIP, FEP }\end{array}$ & NA & AM, D, MI, C, TE \\
\hline HK10-R2 & ND & 16 & 0.25 & 0.25 & WT & WT & - & WT & - & $\begin{array}{l}\text { LVX, GM, CRO, AN, GAT, } \\
\text { F/M, FOS, CIP, FEP }\end{array}$ & & AM, NA, D, Ml, C, TE \\
\hline HK10-R3 & ND & 16 & 0.25 & 0.25 & T119S & WT & - & WT & - & $\begin{array}{l}\text { LVX, GM, CRO, AN, GAT, } \\
\text { F/M, FOS, CIP, FEP }\end{array}$ & NA & AM, D, MI, C, TE \\
\hline HK2-R1 & ND & 4 & 0.25 & 1 & $\begin{array}{l}\text { Insertion of } \\
\quad 7 n t \text { at } \\
+205 \\
\text { (frameshift) }\end{array}$ & WT & - & WT & - & AN, FOS & & $\begin{array}{l}\text { AM, CRO, F/M, NA, } \\
\text { FEP, GM, CIP, LVX, } \\
\text { GAT, D, C, MI, TE }\end{array}$ \\
\hline \multicolumn{13}{|c|}{ Animal isolates } \\
\hline AK297 & ST1326 & 16 & 0.25 & 0.25 & WT & WT & $+\mathrm{WT}$ & WT & - & FEP, AN, CRO & FOS, GM & $\begin{array}{l}\text { D, MI, CIP, LVX, NA, } \\
\text { AM, GAT, C, F/M, TE }\end{array}$ \\
\hline AK298 & ST147 & 8 & 0.25 & 0.12 & Y59stop & WT & - & WT & - & GM, FEP, AN, CRO & & $\begin{array}{c}\text { FOS, D, MI, CIP, LVX, } \\
\text { NA, AM, GAT, C, F/M, } \\
\text { TE }\end{array}$ \\
\hline AK299 & ST45 & 8 & 0.25 & 0.12 & Q122stop & WT & $+\mathrm{WT}$ & WT & - & FOS, AN & FEP, C & $\begin{array}{c}\text { D, MI, CIP, LVX, NA, } \\
\text { AM, CRO, GAT, F/M, } \\
\text { GM, TE }\end{array}$ \\
\hline AK513 & ST37 & 8 & $>128$ & 0.25 & WT & R90G & + WT & $\begin{array}{l}\text { Insertion of } \\
\text { IS3-like } \\
\text { between } \\
+112,+113\end{array}$ & - & FOS, FEP, AN, CRO & GM & $\begin{array}{l}\text { D, MI, CIP, LVX, NA, } \\
\text { AM, GAT, C, F/M, TE }\end{array}$ \\
\hline \multicolumn{13}{|c|}{ Human isolates } \\
\hline HK5 & ST377 & 4 & 0.12 & 16 & $\begin{array}{l}\text { Insertion of } \\
\text { 3nt at } \\
+343\end{array}$ & WT & - & WT & OXA-48 & AN & FOS & $\begin{array}{l}\text { LVX, D, MI, CIP, AM, } \\
\text { NA, GM, CRO, GAT, } \\
\text { FEP, TE, C, F/M }\end{array}$ \\
\hline HK6 & ST16 & 4 & 0.12 & 8 & $1141 \mathrm{~T}$ & $\begin{array}{c}\triangle \mathrm{G} 139^{\mathrm{b}} \\
\text { (frameshift) }\end{array}$ & $+\mathrm{WT}$ & WT & OXA-48 & FOS & & $\begin{array}{l}\text { LVX, D, MI, CIP, AM, } \\
\text { AN, NA, GM, CRO, } \\
\text { GAT, FEP, F/M, TE, C }\end{array}$ \\
\hline
\end{tabular}


TABLE 2 | Continued

\begin{tabular}{|c|c|c|c|c|c|c|c|c|c|c|c|c|}
\hline \multirow{2}{*}{ Isolate } & \multirow{2}{*}{$\begin{array}{l}\text { Sequence } \\
\text { type }\end{array}$} & \multicolumn{3}{|c|}{ MIC (mg/l) } & \multirow[t]{2}{*}{ RamR } & \multirow[t]{2}{*}{ AcrR } & \multirow[t]{2}{*}{ TetA } & \multirow[t]{2}{*}{ MgrB } & \multirow[t]{2}{*}{ Carbapenemase } & \multicolumn{3}{|c|}{ Antimicrobial susceptibility profile } \\
\hline & & Tgc & Col & Ipm & & & & & & $\mathbf{S}$ & $\mathbf{I}$ & $\mathbf{R}$ \\
\hline HK98 & ST147 & 4 & 0.12 & 128 & G180D & WT & - & WT & NDM-1 & & FOS & $\begin{array}{l}\text { D, MI, LVX, CIP, AM, } \\
\text { NA, GM, CRO, GAT, } \\
\text { FEP, TE, AN, C, F/M }\end{array}$ \\
\hline HK156 & ST893 & 4 & 64 & 32 & WT & $\begin{array}{c}\triangle \mathrm{G} 139^{\mathrm{b}} \\
\text { (frameshift) }\end{array}$ & $+W T$ & $\begin{array}{c}\text { Premature } \\
\text { termination by } \\
\text { nonsense } \\
\text { mutation at } \\
\text { nt88 }\end{array}$ & OXA-48 & AN, C & FOS, F/M & $\begin{array}{c}\text { MI, D, CIP, LVX, AM, } \\
\text { NA, GM, CRO, GAT, } \\
\text { FEP, TE }\end{array}$ \\
\hline HK157 & ST2935 & 8 & $>128$ & 64 & $\begin{array}{c}\Delta 12 \mathrm{nt} \\
(\mathrm{a} 205-\mathrm{c} 216), \\
\text { I141T }\end{array}$ & $\begin{array}{c}\Delta 12 \mathrm{nt} \\
(\mathrm{a} 430-\mathrm{g} 441)\end{array}$ & - & $\begin{array}{c}\text { Premature } \\
\text { termination by } \\
\text { nonsense } \\
\text { mutation at } \\
\text { nt88 }\end{array}$ & OXA-48 NDM-5 & FOS & C & $\begin{array}{c}\text { MI, D, CIP, LVX, AM, } \\
\text { NA, GM, CRO, GAT, } \\
\text { FEP, TE, AN, F/M }\end{array}$ \\
\hline
\end{tabular}

WT, wild type; ND, not determined, S, susceptible; I, intermediate; $R$, resistant.

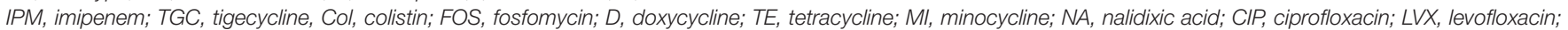
GAT, gatifloxacin, GM, gentamicin; AN, amikacin; AM, ampicillin; CRO, ceftriaxone; FEP, cefepime; C, chloramphenicol; F/M, nitrofurantoin.

${ }^{a} \Delta$ ramR locus, not amplifiable with all primers used in this study.

${ }^{b}$ Deletion of one of the guanines at positions 134-139.

wild-type RpsJ and TetA (if present). The ramR amplicons were obtained for 17 out of 18 TGC-NSKP isolates with the exception of isolate AK294, which did not yield any PCR product using the two pairs of primers used in this study. Eleven out of eighteen TGC-NSKP isolates (61\%) ( $n=3$ animal, 4 human, and 4 in vitro selected mutants) revealed mutated/altered $\mathrm{ramR}$ gene. The observed RamR alterations included premature termination by nonsense mutations at amino acid positions 59 [TAC $(\mathrm{Y})>\mathrm{TAA}]$ and 122 [CAG (Q) > TAG]; a 12-nt deletion at positions 205-216; a 3-nt (AGC, serine) insertion at position +343 (between +342 , +343); substitutions A19V, G180D, and I141T found among human and animal TGC-NSKP isolates and frameshift mutation due to insertion of $7 \mathrm{nt}$ at position +205 ; and substitutions L44Q, $\mathrm{A} 28 \mathrm{~T}+\mathrm{R} 114 \mathrm{~L}$, and $\mathrm{T} 119 \mathrm{~S}$ observed among in vitro selected mutants. Moreover, AcrR alterations were observed among seven (38\%) TGC-NSKP isolates (three animal isolates, three human isolates, and one in vitro selected mutant). A frameshift mutation resulting from deletion of one of the six guanines at positions 134-139 was the most common AcrR alteration identified among both animal $(n=1)$ and human isolates $(n=2)$. AcrR R90G substitution was found among two animal isolates, both of which carried a wild-type RamR protein. Also a frameshift mutation resulting from 14-nt deletions (g58-t71) was detected in one in vitro selected mutant (TGC MIC $=32 \mathrm{mg} / \mathrm{l}$ ) carrying a wild-type RamR. One TGC-NSKP human isolate coharbored two 12-nt deletion in $\operatorname{ramR}$ (a205-c216) and acrR (a430g441) genes (Table 2). Among seven colistin-resistant isolates, five harbored inactivated/mutated MgrB due to premature termination by nonsense mutations, insertion of IS elements, and frameshift mutation as shown in Table 2. The genes encoding for carbapenemases were detected in all IPM-resistant human isolates, with three isolates harboring bla $a_{\mathrm{OXA}-48}$, one carrying $b l a_{\mathrm{NDM}-1}$, and one co-harboring $b l a_{\mathrm{OXA}-48}$ and $b l a_{\mathrm{NDM}-5}$. Two TGC-RKP isolates with TGC MIC = $16 \mathrm{mg} / \mathrm{l}$ carried wild-type RamR and AcrR proteins (Table 2).

\section{Expression Levels of the AcrAB Pump}

To see if TGC non-susceptibility was linked to overexpression of $A c r A B$ pump, the expression level of $a c r B$ gene was quantified by RT-qPCR analysis. A constitutively expressed housekeeping gene $r p s L$ was used as a control and the TGC-susceptible clinical strain HK10 as a reference for the data analysis of animal and human isolates. For the in vitro selected mutants, the expression level was compared with that of their TGC$\mathrm{S}$ parental strains. The in vitro selected TGC-NSKP isolates showed the highest expression levels (18- to 146-fold) among the studied isolates. Since the expression level of $a c r B$ in all three TGC-S parental isolates (K88-S, K10-S, and K2-S) were similar (representing with similar $\Delta_{\mathrm{CT}}$ ), there was no significant difference when $a c r B$ expression of in vitro selected mutants was compared with that of parental TGC-S or with the TGC$S$ clinical isolate HK10-S. The TGC-RKP isolates of animal and human origins displayed levels of $\operatorname{acr} B$ expression that were 5- to 62-fold and 2- to 26-fold higher than those of control HK10, respectively (Figure 1). These data support the hypothesis that increased expression of $\operatorname{acr} B$ is associated with increased MICs of TGC.

\section{DISCUSSION}

Multidrug-resistant isolates of $K$. pneumoniae have emerged in past years as results of wide application of antibacterial agents in both human and veterinary medicine. Indeed, consumption of human antibiotics in veterinary practices has been blamed for contributing to the magnitude of current antibiotic resistance crisis. Emergence of antibiotic resistance among commensal bacteria propagated in food animals, posing a great challenge for human health since they have the potential to reach human hosts through the food chain or transmit their mobile resistance 


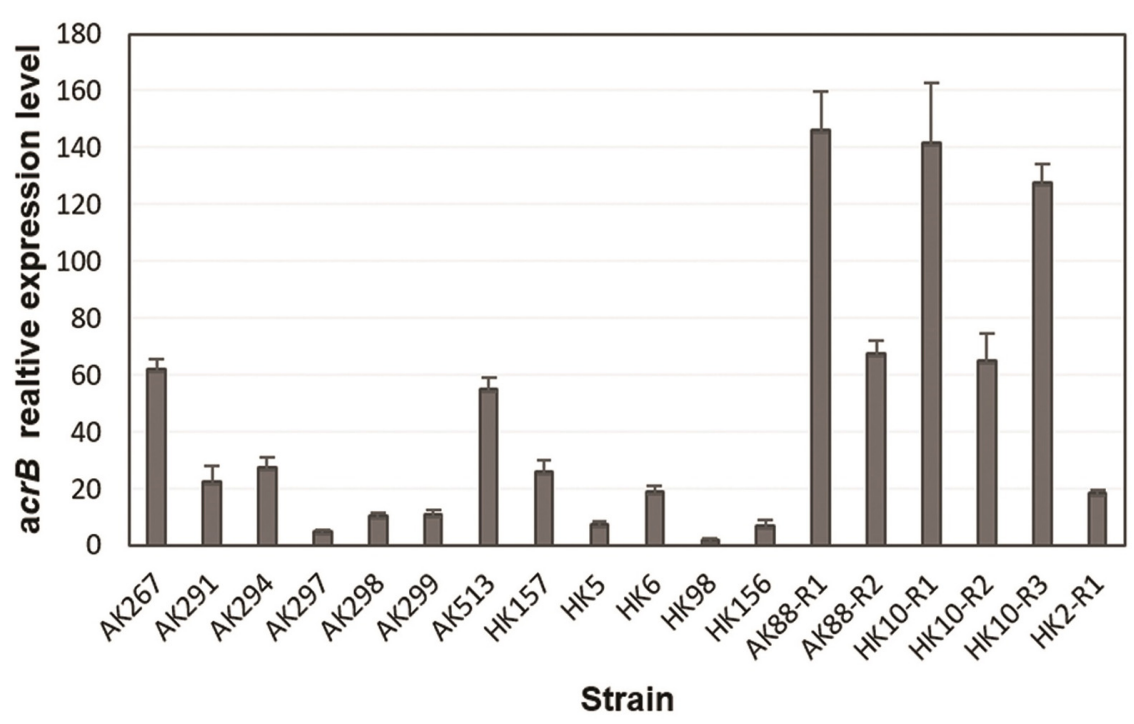

FIGURE 1 | Relative expression of acrB gene from tigecycline (TGC)-non-susceptible isolates of Klebsiella pneumoniae from different origins determined by RT-qPCR.

elements to human pathogens (Founou et al., 2016). Resistance of $K$. pneumoniae to various classes of antibiotics can be mediated by a variety of mechanisms, among which extrusion of antibiotics using efflux machineries such as AcrAB has been well-studied. Due to its broad substrate range property, AcrAB pump has been found to mediate resistance to a different families of antibiotics such as penicillins, cephalosporins, fluoroquinolones, macrolides, chloramphenicol, and tetracyclines (Xu et al., 2021). In the current study, screening among commensal bacteria of broiler chickens revealed six TGC-RKP isolates that were also resistant to quinolones, chloramphenicol, nitrofurantoin, and other tetracyclines. There are currently no TGC-containing products authorized for the veterinary use in Iran, and it is likely that selective pressure caused by application of other antibiotics such as older tetracyclines, florfenicol, or enrofloxacin may contribute to TGC non-susceptibility in animal commensal bacterial isolates. In a recent study from China, five TGC-RKP isolates that all belonged to ST1 were identified from the fecal samples of healthy chickens. Resistance to TGC as well as other antibiotics in these isolates was found to be mediated by a novel plasmid-mediated RND efflux pump gene cluster, designated tmexCD1-toprJ1 (Lv et al., 2020). Li et al. also reported isolation of TGC-R Klebsiella aerogenes (TGC MIC = $32 \mathrm{mg} / \mathrm{l}$ ) from the feces of a chicken farm, which contained the $b l a_{\mathrm{NDM}-9}$ and tet(A) variant genes (Li et al., 2021). Analyzing the genetic relatedness of TGC-NSKP isolates of both origins using MLST revealed high genetic diversity among the isolates with 12 TGCNSKP isolates belonging to 10 distinct STs, among which ST147 was found as a common type between two human and animal isolates. Most of the animal isolates belonged to STs, which are commonly reported from different human infections including ST11 (Gu et al., 2018), ST37 (Zhao et al., 2019), ST15, and ST147 (Yan et al., 2015; Chen et al., 2020). Co-resistance to TGC and colistin was observed among animal isolates belonging to ST11,
ST37, and ST15. Other studies have also reported occurrence of TGC and colistin co-resistance among $K$. pneumoniae isolates belonging to ST37 and ST11 (Taniguchi et al., 2017; Xu et al., 2020). A recent study also reported detection of colistin and TGC-RKP (ST29) in municipal wastewater influents from Japan (Hayashi et al., 2021). All human TGC-NSKP isolates showed co-resistance to carbapenems and carried genes encoding for two different carbapenemases. The isolate HK157 belonging to ST2935 revealed co-resistance to three last-resort antibiotics and co-harbored a truncated $m g r B$ gene, mutant $r a m R$ and $a c r R$ genes, and $b l a_{\mathrm{OXA}}-48$ and $b l a_{\mathrm{NDM}-5}$ carbapenemase genes. This is the first study reporting detection of $b l a_{\mathrm{NDM}-5}$ among clinical CRKP isolates from Iran.

Analysis of the genes, AcrR (AcrAB repressor) and the RamR regulator, revealed inactivating genetic alterations in both proteins. In $83 \%$ of isolates ( $n=15$ out of 18 ) mutation/deletions in $\operatorname{ramR}(n=8$ isolates) or $\operatorname{acrR}(n=4)$ or both $(n=3)$ was identified. The $\operatorname{ram} R$ was not detected in one isolate (AK294) using two pairs of primers used for amplification of the $r a m R$ gene, suggesting deletion of $r a m R$ locus in this isolate. In two animal isolates, RamR was truncated due to nonsense mutation, which resulted in production of 58 and 121 amino acid long proteins instead of wild-type protein with 193 amino acids. The RamR Q122 stop substitution has been previously reported among TGC-NSKP isolates by several other studies presenting this alteration as a common resistance mechanism among K. pneumoniae isolates (He et al., 2015; Chiu et al., 2017b; Li et al., 2017; Park et al., 2020). In addition to two previously reported RamR A19V and L44Q mutations (reported as L44R) (Chiu et al., 2017b), five novel amino acid substitutions, including I141T, G180D, A28T, R114L, and T119S, were identified, with the latter three changes being detected after resistance induction in in vitro selected mutants. The A19V substitution, which was also predicted by the PROVEAN tool to be a neutral change, 
has been previously demonstrated to have no effect on the TGC MICs (Chiu et al., 2017b). While RamR A28T, R114L, and G180D substitutions were predicted by the PROVEAN tool to have a deleterious impact on protein structure (PEOVEAN scores -3.6, -4 , and -6.8 , respectively), the I141T and T119S substitutions (with PEOVEAN scores 0.21 and -2.44 (very close to prediction cutoff $=-2.5$ ), respectively) were predicted to be neutral changes. An isolate-carrying RamR I141T substitution co-harbored an AcrR inactivating mutation (frameshift), indicating that AcrR alteration might be the main mediator of TGC non-susceptibility in this bacterium. However, the exact role of these novel mutations in elevation of TGC MICs requires further studies by confirmatory assays. In one in vitro selected mutant and a clinical TGC-R isolate nucleotide insertion (7nt) and deletion (12 nt) at position +205 was observed, respectively, indicating that this position is more prone to alterations upon resistance development. Among the 18 TGC-NSKP, mutations/deletions within AcrR were detected among seven isolates from which six carried a wild-type RamR or RamR substitutions, which were predicted or previously demonstrated to be neutral changes. The novel AcrR R90G substitution detected in two TGC-R animal isolates belonging to ST37 was predicted by the PROVEAN tool to have a deleterious effect on the functionality of the protein (PROVEAN score $=-5.9$ ). The AcrR frameshift mutation mediated by deletion of one of the guanines at positions 134-139 was detected in three TGC-NSKP isolates of both origins that belonged to different STs representing this region as a mutation-prone position within $a c r R$ gene. In two TGCRKP isolates with overexpressed AcrB (AK297 and HK10-R2, TGC MIC $=16 \mathrm{mg} / \mathrm{l}$ ), no alteration was identified in any of the studied genes, indicating that alterations in promoter region of the studied genes or other loci (marR or soxR; Bratu et al., 2009; Veleba and Schneiders, 2012) are probably involved in resistance development in these isolates. Gene expression analysis revealed an association between overexpression of AcrAB efflux pump and TGC non-susceptibility. However, there was no correlation between the TGC MICs and the level of $a c r B$ expression, as some isolates with similar MICs exhibited different expression levels. This suggests the possibility of contribution of other resistance mechanisms to increased TGC MICs.

\section{CONCLUSION}

A combination of genetic alterations in AcrR and RamR mediated the overexpression of AcrAB efflux pump and

\section{REFERENCES}

Bartha, N. A., Sók, J., Urbán, E., and Nagy, E. (2011). Investigation of the prevalence of tet $Q$, tet $X$ and tet $X 1$ genes in Bacteroides strains with elevated tigecycline minimum inhibitory concentrations. Int. J. Antimicrob. Agents 38, 522-525. doi: 10.1016/j.ijantimicag.2011.07.010

Beabout, K., Hammerstrom, T. G., Perez, A. M., Magalhães, B. F., Prater, A. G., Clements, T. P., et al. (2015). The ribosomal S10 protein is a general target for decreased tigecycline susceptibility. Antimicrob. Agents Chemother. 59, 5561-5566. doi: 10.1128/aac.00547-15 subsequently TGC non-susceptibility among the majority of human, animal, and in vitro selected mutants studied in this work. The most worrisome finding in our study was detection of multidrug-resistant isolates with co-resistance to two last-resort human antibiotics (TGC and colistin) belonging to sequence types commonly implicated in human infections (ST11, ST37, and ST15) among commensal bacteria of food animals. This can be considered a great threat to human health due to probability of transmission of these bacteria to humans through the food chain or direct contact. Acquiring carbapenem resistance among these isolates would be the most troublesome event. While the TGC exposure history among the human isolates was not clear, emergence of TGC resistance among animal isolates (which are not exposed to TGC) is an issue of great concern. It is speculated that selective pressure caused by other antimicrobials in particular tetracycline families (as one of the most used antimicrobials in food animals) may have contributed to increased TGC MICs in these isolates probably through the overexpression of AcrAB efflux pump. Therefore, immediate actions need to be taken to restrict/minimize the use of human antibiotics (at least not as growth promoters) in food animals to prevent the emergence and dissemination of antibiotic-resistant bacteria through the food chain.

\section{DATA AVAILABILITY STATEMENT}

Publicly available datasets were analyzed in this study. This data can be found here: GenBank; accession numbers MW653710MW653741.

\section{AUTHOR CONTRIBUTIONS}

MM performed the experiments, analyzed the experiment data, and drafted the manuscript. $\mathrm{MH}$ and HM designed the experiments, analyzed the experiment data, and wrote the manuscript. All authors read and approved the final manuscript.

\section{FUNDING}

This study was supported by the University of Tabriz.

Bratu, S., Landman, D., George, A., Salvani, J., and Quale, J. (2009). Correlation of the expression of acrB and the regulatory genes marA, soxS and ramA with antimicrobial resistance in clinical isolates of Klebsiella pneumoniae endemic to New York City. J. Antimicrob. Chemother. 64, 278-283. doi: 10.1093/jac/ dkp186

Cannatelli, A., D’Andrea, M. M., Giani, T., Di Pilato, V., Arena, F., Ambretti, S., et al. (2013). In vivo emergence of colistin resistance in Klebsiella pneumoniae producing KPC-type carbapenemases mediated by insertional inactivation of the PhoQ/PhoP mgrB regulator. Antimicrob. Agents Chemother. 7, 5521-5526. doi: 10.1128/AAC.01480-13 
Chen, D., Li, H., Zhao, Y., Qiu, Y., Xiao, L., He, H., et al. (2020). Characterization of carbapenem-resistant Klebsiella pneumoniae in a tertiary hospital in Fuzhou, China. J. Appl. Microbiol. 129, 1220-1226. doi: 10.1111/jam. 14700

Chiu, S.-K., Chan, M.-C., Huang, L.-Y., Lin, Y.-T., Lin, J.-C., Lu, P.-L., et al. (2017a). Tigecycline resistance among carbapenem-resistant Klebsiella pneumoniae: clinical characteristics and expression levels of efflux pump genes. PLoS One 12:e0175140. doi: 10.1371/journal.pone.017 5140

Chiu, S.-K., Huang, L.-Y., Chen, H., Tsai, Y.-K., Liou, C.-H., Lin, J.-C., et al. (2017b). Roles of ramR and tet (A) mutations in conferring tigecycline resistance in carbapenem-resistant Klebsiella pneumoniae clinical isolates. Antimicrob. Agents Chemother. 61, e00391-17.

Choi, Y., and Chan, A. P. (2015). PROVEAN web server: a tool to predict the functional effect of amino acid substitutions and indels. Bioinformatics 31, 2745-2747. doi: 10.1093/bioinformatics/btv195

Deng, M., Zhu, M.-H., Li, J.-J., Bi, S., Sheng, Z.-K., Hu, F.-S., et al. (2014). Molecular epidemiology and mechanisms of tigecycline resistance in clinical isolates of Acinetobacter baumannii from a Chinese university hospital. Antimicrob. Agents Chemother. 58, 297-303. doi: 10.1128/aac. 01727-13

Diancourt, L., Passet, V., Verhoef, J., Grimont, P. A., and Brisse, S. (2005). Multilocus sequence typing of Klebsiella pneumoniae nosocomial isolates. J. Clin. Microbiol. 43, 4178-4182. doi: 10.1128/jcm.43.8.4178-4182. 2005

Du, X., He, F., Shi, Q., Zhao, F., Xu, J., Fu, Y., et al. (2018). The rapid emergence of tigecycline resistance in blaKPC-2 harboring Klebsiella pneumoniae, as mediated in vivo by mutation in tetA during tigecycline treatment. Front. Microbiol. 9:648. doi: 10.3389/fmicb.2018.00648

Founou, L. L., Founou, R. C., and Essack, S. Y. (2016). Antibiotic resistance in the food chain: a developing country-perspective. Front. Microbiol. 7:1881. doi: $10.3389 /$ fmicb.2016.01881

Gu, D., Dong, N., Zheng, Z., Lin, D., Huang, M., Wang, L., et al. (2018). A fatal outbreak of ST11 carbapenem-resistant hypervirulent Klebsiella pneumoniae in a Chinese hospital: a molecular epidemiological study. Lancet Infect. Dis. 18, 37-46. doi: 10.1016/s1473-3099(17)30489-9

Haeili, M., Javani, A., Moradi, J., Jafari, Z., Feizabadi, M. M., and Babaei, E. (2017). MgrB alterations mediate colistin resistance in Klebsiella pneumoniae isolates from Iran. Front. Microbiol. 8:2470. doi: 10.3389/fmicb.2017.02470

Hayashi, W., Iimura, M., Soga, E., Koide, S., Izumi, K., Yoshida, S., et al. (2021). Presence of colistin-and tigecycline-resistant Klebsiella pneumoniae ST29 in municipal wastewater influents in Japan. Microb. Drug Resist. doi: 10.1089/mdr. 2020.0514 [Epub ahead of print].

He, F., Fu, Y., Chen, Q., Ruan, Z., Hua, X., Zhou, H., et al. (2015). Tigecycline susceptibility and the role of efflux pumps in tigecycline resistance in KPCproducing Klebsiella pneumoniae. PLoS One 10:e0119064. doi: 10.1371/journal. pone.0119064

Hentschke, M., Wolters, M., Sobottka, I., Rohde, H., and Aepfelbacher, M. (2010). ramR mutations in clinical isolates of Klebsiella pneumoniae with reduced susceptibility to tigecycline. Antimicrob. Agents Chemother. 54, 2720-2723. doi: 10.1128/aac.00085-10

Jafari, Z., Harati, A. A., Haeili, M., Kardan-Yamchi, J., Jafari, S., Jabalameli, F., et al. (2019). Molecular epidemiology and drug resistance pattern of carbapenemresistant Klebsiella pneumoniae isolates from Iran. Microb. Drug Resist. 25, 336-343.

Li, R., Han, Y., Zhou, Y., Du, Z., Wu, H., Wang, J., et al. (2017). Tigecycline susceptibility and molecular resistance mechanisms among clinical Klebsiella pneumoniae strains isolated during non-tigecycline treatment. Microb. Drug Resist. 23, 139-146. doi: 10.1089/mdr.2015.0258

Li, Y., Wang, Q., Xiao, X., Li, R., and Wang, Z. (2021). Emergence of blaNDM-9-bearing tigecycline resistant Klebsiella aerogenes of chicken origin. J. Glob. Antimicrob. Resist. 26, 66-68. doi: 10.1016/j.jgar.2021. 04.028

Livermore, D. M. (2005). Tigecycline: what is it, and where should it be used? J. Antimicrob. Chemother. 56, 611-614. doi: 10.1093/jac/ dki291

Lv, L., Wan, M., Wang, C., Gao, X., Yang, Q., Partridge, S. R., et al. (2020). Emergence of a plasmid-encoded resistance-nodulation-division efflux pump conferring resistance to multiple drugs, including tigecycline, in Klebsiella pneumoniae. mBio 11, e02930-19.

Mahon, C. R., Lehman, D. C., and Manuselis, G. (2018). Textbook of Diagnostic Microbiology-E-Book. Amsterdam: Elsevier Health Sciences.

Moore, I. F., Hughes, D. W., and Wright, G. D. (2005). Tigecycline is modified by the flavin-dependent monooxygenase TetX. Biochemistry 44, 11829-11835. doi: 10.1021/bi0506066

Park, Y., Choi, Q., Kwon, G. C., and Koo, S. H. (2020). Molecular epidemiology and mechanisms of tigecycline resistance in carbapenem-resistant Klebsiella pneumoniae isolates. J. Clin. Lab. Anal. 34:e23506.

Pishnian, Z., Haeili, M., and Feizi, A. (2019). Prevalence and molecular determinants of colistin resistance among commensal Enterobacteriaceae isolated from poultry in northwest of Iran. Gut pathog. 11, 1-8.

Pitout, J. D., Nordmann, P., and Poirel, L. (2015). Carbapenemase-producing Klebsiella pneumoniae, a key pathogen set for global nosocomial dominance. Antimicrob. Agents Chemother. 59, 5873-5884. doi: 10.1128/aac.010 19-15

Poirel, L., Walsh, T. R., Cuvillier, V., and Nordmann, P. (2011). Multiplex PCR for detection of acquired carbapenemase genes. Diagn. Microbiol. Infect. Dis. 70, 119-123. doi: 10.1016/j.diagmicrobio.2010. 12.002

Renteria, M., Biedenbach, D., Bouchillon, S., Hoban, D., Raghubir, N., and Sajben, P. (2014). In vitro activity of tigecycline and comparators against carbapenemresistant Enterobacteriaceae in Africa-Middle East countries: TEST 20072012. J. Glob. Antimicrob. Resist. 2, 179-182. doi: 10.1016/j.jgar.2014. 03.002

Rosenblum, R., Khan, E., Gonzalez, G., Hasan, R., and Schneiders, T. (2011). Genetic regulation of the ramA locus and its expression in clinical isolates of Klebsiella pneumoniae. Int. J. Antimicrob. Agents 38, 39-45. doi: 10.1016/j. ijantimicag.2011.02.012

Sader, H. S., Castanheira, M., Flamm, R. K., Mendes, R. E., Farrell, D. J., and Jones, R. N. (2015). Tigecycline activity tested against carbapenem-resistant Enterobacteriaceae from 18 European nations: results from the SENTRY surveillance program (2010-2013). Diagn. Microbiol. Infect. Dis. 83, 183-186. doi: 10.1016/j.diagmicrobio.2015.06.011

Spanu, T., De Angelis, G., Cipriani, M., Pedruzzi, B., D’Inzeo, T., Cataldo, M. A., et al. (2012). In vivo emergence of tigecycline resistance in multidrug-resistant Klebsiella pneumoniae and Escherichia coli. Antimicrob. Agents Chemother. 56, 4516-4518.

Taniguchi, Y., Maeyama, Y., Ohsaki, Y., Hayashi, W., Osaka, S., Koide, S., et al. (2017). Co-resistance to colistin and tigecycline by disrupting mgrB and ramR with IS insertions in a canine Klebsiella pneumoniae ST37 isolate producing SHV-12, DHA-1 and FosA3. Int. J. Antimicrob. Agents 50, 697-698. doi: 10. 1016/j.ijantimicag.2017.09.011

Van, T. T. H., Yidana, Z., Smooker, P. M., and Coloe, P. J. (2020). Antibiotic use in food animals worldwide, with a focus on Africa: pluses and minuses. J. Glob. Antimicrob. Resist. 20, 170-177. doi: 10.1016/j.jgar.2019. 07.031

Veleba, M., and Schneiders, T. (2012). Tigecycline resistance can occur independently of the ramA gene in Klebsiella pneumoniae. Antimicrob. Agents Chemother. 56, 4466-4467. doi: 10.1128/aac. 06224-11

$\mathrm{Xu}$, J., Zhao, Z., Ge, Y., and He, F. (2020). Rapid emergence of a pandrug-resistant Klebsiella pneumoniae ST11 isolate in an inpatient in a teaching hospital in China after treatment with multiple broadspectrum antibiotics. Infect. Drug Resist. 13, 799-804. doi: 10.2147/idr.s2 43334

Xu, Q., Sheng, Z., Hao, M., Jiang, J., Ye, M., Chen, Y., et al. (2021). RamA upregulates multidrug resistance efflux pumps AcrAB and OqxAB in Klebsiella pneumoniae. Int. J. Antimicrob. Agents 57:106251. doi: 10.1016/j.ijantimicag. 2020.106251

Yan, J., Wang, M., Zheng, P., Tsai, L., and Wu, J. (2015). Associations of the major international high-risk resistant clones and virulent clones with specific ompK36 allele groups in Klebsiella pneumoniae in Taiwan. New Microbes New Infect. 5, 1-4. doi: 10.1016/j.nmni.2015. 01.002

Zhao, Y., Zhang, X., Torres, V. V. L., Liu, H., Rocker, A., Zhang, Y., et al. (2019). An outbreak of carbapenem-resistant and hypervirulent Klebsiella 
pneumoniae in an intensive care unit of a major teaching hospital in Wenzhou, China. Front. Public Health 7:229. doi: 10.3389/fpubh.2019. 00229

Conflict of Interest: The authors declare that the research was conducted in the absence of any commercial or financial relationships that could be construed as a potential conflict of interest.

Publisher's Note: All claims expressed in this article are solely those of the authors and do not necessarily represent those of their affiliated organizations, or those of the publisher, the editors and the reviewers. Any product that may be evaluated in this article, or claim that may be made by its manufacturer, is not guaranteed or endorsed by the publisher.

Copyright $\odot 2021$ Moghimi, Haeili and Mohajjel Shoja. This is an open-access article distributed under the terms of the Creative Commons Attribution License (CC BY). The use, distribution or reproduction in other forums is permitted, provided the original author(s) and the copyright owner(s) are credited and that the original publication in this journal is cited, in accordance with accepted academic practice. No use, distribution or reproduction is permitted which does not comply with these terms. 